\title{
Microstreaming inside model cells induced by ultrasound and microbubbles
}

Valerio Pereno, Junjun Lei, Dario Carugo, and Eleanor Stride ${ }^{*}$

Dr. V. Pereno, Prof. E. Stride

Institute of Biomedical Engineering, Department of Engineering Science, University of Oxford, Oxford OX3 7DQ, U.K.

Email: eleanor.stride@eng.ox.ac.uk

Dr. J. Lei

State Key Laboratory of Precision Electronic Manufacturing Technology and Equipment, Guangdong University of Technology, Guangzhou 510006, China

Dr. D. Carugo

Faculty of Engineering and Physical Sciences and Institute for Life Sciences, Department of Mechanical Engineering, University of Southampton, Southampton SO17 1BJ, U.K.

\begin{abstract}
Studies on the bioeffects produced by ultrasound and microbubbles have focused primarily on transport in bulk tissue, drug uptake by individual cells, and disruption of biological membranes. Relatively little is known about the physical perturbations and fluid dynamics of the intracellular environment during ultrasound exposure. To investigate this, a custom acoustofluidic chamber was designed to expose model cells, in the form of giant unilamellar vesicles, to ultrasound and microbubbles. The motion of fluorescent tracer beads within the lumen of the vesicles was tracked during exposure to laminar flow $\left(\sim 1 \mathrm{~mm} \mathrm{~s}^{-1}\right)$, ultrasound (1MHz, $\sim 150 \mathrm{kPa}, 60 \mathrm{~s})$, and phospholipid coated microbubbles, alone and in combination. To decouple the effects of fluid flow and ultrasound exposure, the system was also modelled numerically using boundary-driven streaming field equations. Both the experimental and
\end{abstract}


numerical results indicate that all conditions produced internal streaming within the vesicles. Ultrasound alone produced an average bead velocity of $6.5 \pm 1.3 \mu \mathrm{m} / \mathrm{s}$, which increased to $8.5 \pm 3.8 \mu \mathrm{m} / \mathrm{s}$ in the presence of microbubbles compared to $12 \pm 0.12 \mu \mathrm{m} / \mathrm{s}$ under laminar flow. Further research on intracellular forces in mammalian cells and the associated biological effects in vitro and in vivo are required to fully determine the implications for safety and/or therapy.

Keywords: microstreaming; intracellular streaming; microbubbles; ultrasound; drug delivery.

\section{Introduction}

Gas-filled microbubbles stabilised by phospholipid, denatured protein, or polymer coatings are widely used in medical imaging as ultrasound contrast agents due to their high echogenicity. More recently, their therapeutic potential has been investigated in the context of targeted drug delivery, with emphasis on transient permeabilisation of the cell membrane - a phenomenon commonly referred to as sonoporation.

When exposed to an ultrasound field, microbubbles expand and contract radially at a frequency and amplitude determined by those of the ultrasound wave ${ }^{1}$. When these volumetric oscillations occur proximal to the cell plasma membrane, the resulting tensile, compressive, and shear forces associated with microbubble dynamics lead to various bioeffects, which may include activation of mechanosensitive channels, poration, or irreversible rupture of the cell membrane ${ }^{2}$. Transient formation of transmembrane pores has been observed in vitro ${ }^{4,5,6}$, and has been shown to promote targeted uptake of therapeutic molecules ${ }^{7}$. However, it remains unclear whether physical alterations of the cell membrane are induced directly by the forces imparted by cavitating microbubbles themselves, by thermal or chemical effects, or as a result of the shear stress caused by cavitation microstreaming. 
Microstreaming is produced as a result of the bubble oscillations ${ }^{3}$. Attenuation of this motion in the surrounding viscous medium, generates a steady flow and high fluid velocity gradients in the bubble's immediate vicinity. Particle velocimetry experiments have shown that microstreaming flows around oscillating microbubbles can reach velocities of the order of a few millimetres per second, at distances greater than a millimetre away from the microbubbles themselves $^{3}$, while imparting shear stresses of the order of Pascals on nearby surfaces ${ }^{3,8,9,10}$. The resulting membrane strain in the presence of microstreaming has been hypothesized to cause temporary permeabilisation ${ }^{11}$ and to enhance intracellular uptake of therapeutic compounds. Ultrasound and microbubble-mediated membrane effects have therefore received significant attention in drug delivery. There have been numerous studies on plasma membranes which report poration ${ }^{5,12,13}$, fluidisation ${ }^{14-16}$, membrane surface divergence ${ }^{3}$, and changes in the molecular arrangement of membrane constituents ${ }^{17}$. However, only a limited body of work has focused on shear force transmission from the extracellular to the intracellular compartments.

Investigations on the intracellular effects of ultrasound exposure have primarily focused on plant cells. Although the structure and function of plant cells differ greatly from mammalian cells, they serve as a useful model to elucidate the physical mechanisms that underpin the dynamics of intracellular structures under ultrasound exposure. Previous studies have described "churning" of intracellular organelles at varying acoustic pressures and frequencies, and have reported chromosomal abnormalities ${ }^{18}$, reversible suppression of nucleic acids and protein synthesis, reduction in mitotic index ${ }^{19}$, and changes in the internal structure of mitochondria ${ }^{20}$. At low acoustic pressures, the effects were reported to be largely reversible and mainly involved organelle accumulation and translation. ${ }^{21}$ Zhong et al. ${ }^{13}$ have also shown that the effects of cavitation are not restricted to the cell membrane, but extend to the intracellular milieu as a result of disruption of the interconnected network of subcellular cytoskeletal filaments. Hussein et al. have shown that exposure to ultrasound and microbubbles 
can promote release of intracellular material from the cytoplasm, lysosomes and endosomes; ${ }^{22}$ whilst Yang et al. have shown that membrane repair following sonoporation may be related to lysosomal stimulation. ${ }^{23}$

To the best of the authors' knowledge, however, no previous study has investigated intracellular movements resulting from exposure to therapeutically relevant conditions involving ultrasound and microbubbles. In the context of microbubble-mediated ultrasound drug delivery, intracellular reorganization and the enhancement or inhibition of intracellular transport may potentially have significant implications for drug bioavailability, efficacy, and toxicity ${ }^{24}$. More broadly, movement of intracellular fluid and organelles plays an important role in cellular functions such as endocytosis ${ }^{25}$, and may influence processes that rely on intracellular transfer and balance of ions. For instance, the membrane repair process involved in transient sonoporation has been hypothesised to rely on the recruitment and exocytosis of lipid vesicles that fuse with the plasma membrane to reseal pores at the rupture site ${ }^{26}$. Moreover, modification or interruption of intracellular trafficking by exposure to ultrasound may have safety repercussions, as the impairment of vesicular transport is implicated in various diseases including cancer $^{27}$, diabetes ${ }^{28}$, heart disease ${ }^{29}$, and also in immunological ${ }^{30}$, neurological ${ }^{31}$, and developmental disorders. ${ }^{32}$.

In order to investigate the impact of ultrasound and microbubbles on internal fluid motion, both numerical and experimental techniques were employed in this study. As the primary purpose was to determine whether or not intra-luminal streaming could be induced, rather than the biological consequences, a simplified cell model was utilized in preference to live cells. It was hoped that this would improve both reproducibility and reliable quantification of flow velocities. Giant unilamellar vesicles were selected, as they have been successfully used in earlier investigations of membrane mechanotransduction under shear flow ${ }^{33-35}$. 
In the first part of the study, phospholipid vesicles formed using a mixture of 1,2-dioleoyl-snglycero-3-phosphocholine (DOPC) and cholesterol (natural constituents of plasma membranes) and containing an aqueous solution of sucrose with $500 \mathrm{~nm}$ fluorescent beads, were employed to experimentally investigate shear transmission at the cell membrane. Vesicles were exposed to constant unidirectional flow, ultrasound, and microbubbles, alone and in combination (Figure 1A), and the dynamics of intra-vesicle flow-tracer beads was quantified using particle tracking. In the second part of the study, intra-vesicular fluid flow patterns were evaluated numerically upon exposure to (i) acoustic excitation and (ii) continuous flow in a microfluidic channel by solving boundary-driven streaming field equations.

\section{Results and Discussion}

Experimental results

No exposure

When no excitation was applied, the vesicles maintained their morphology and exhibited minimal fluctuations of the lipid membrane, known as flickering, as previously reported. ${ }^{36-38}$ While most latex beads appeared as individual particles, some clustering was observed. Beads moved within the vesicles' lumen in a random fashion, typical of Brownian motion, and no mean displacement was detected (Figure $1 \mathrm{~B}$ ). The average bead velocity was measured as 0.8 $\pm 0.2 \mu \mathrm{m} / \mathrm{s}$ (Figure $1 \mathrm{C}$ ). 
A

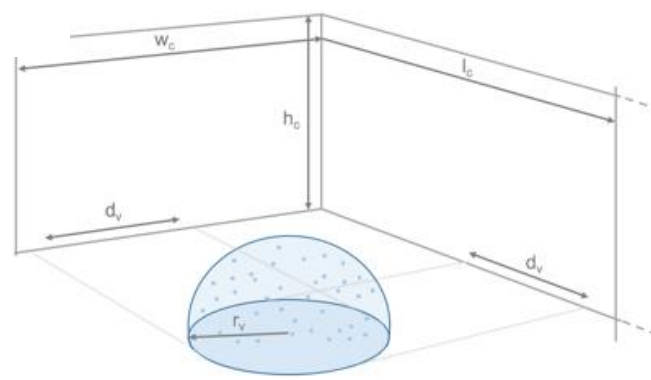

Ultrasound

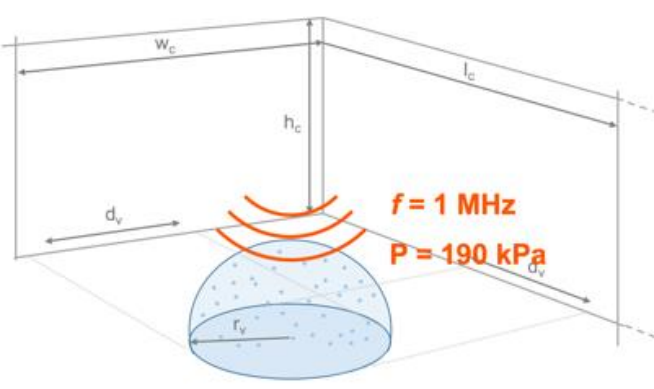

B

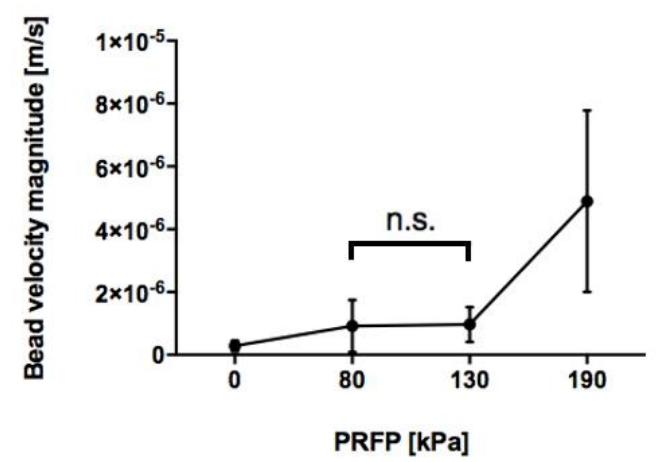

Unidirectional flow

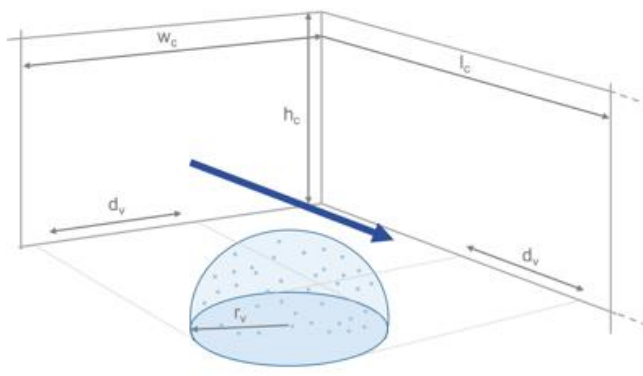

Ultrasound and microbubbles

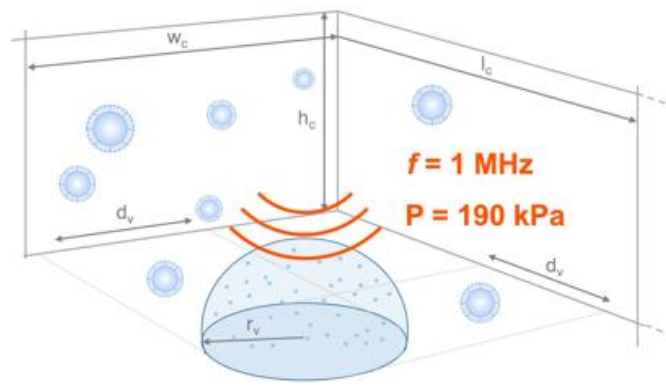

C

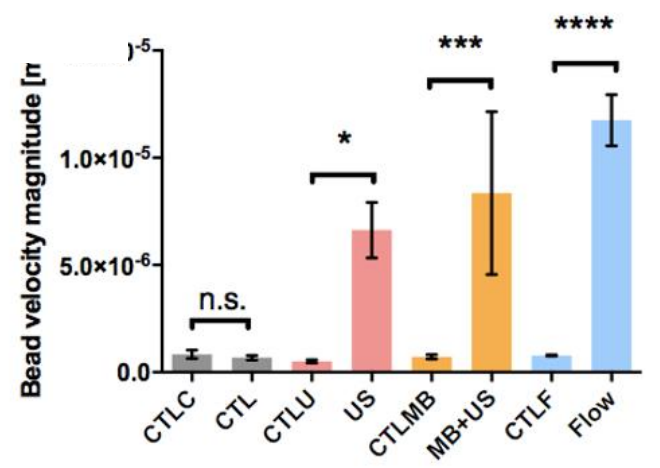

Figure 1 - A. Vesicle exposure conditions. In the control case, vesicles were left to stand for one minute while being imaged with no excitation applied. In the constant flow case, vesicles were subject to a steady flow indicated by the arrow. In the case of ultrasound exposure, vesicles were exposed to a sinusoidal acoustic pressure wave with $190 \mathrm{kPa}$ peak-negative pressure, at $1 \mathrm{MHz}$ frequency (continuous wave). In the presence of microbubbles and ultrasound, DSPC:PEG 9:1 microbubbles were injected to achieve a final concentration of $1 \times 10^{6} \mathrm{MBs} / \mathrm{ml}$ and were immediately exposed to ultrasound in the same conditions as those 
used in the ultrasound-only case. In every condition, vesicles were stimulated for one minute. $d_{v}$ represents the diameter of the vesicle, $w_{c}$ represents the width of the chamber $(12 \mathrm{~mm})$, and $L_{c}$ represents the length of the chamber $(60 \mathrm{~mm})$. B. Luminal bead velocity during exposure to ultrasound alone, at varying ultrasound acoustic pressures C. Average bead velocity magnitude within the vesicle lumen as a result of varying exposure conditions (CTL/C - Control; US/U Ultrasound; MB - microbubble; F - flow). Error bars represent standard deviation for different vesicles exposed to the same conditions. Statistical significance was first tested using a oneway ANOVA on the bead velocities during exposure $(\mathrm{p}<0.01)$, and a Tukey-Cramer post-hoc was used to compare exposure conditions to their respective controls.

\section{Ultrasound exposure}

Upon exposure to ultrasound, fluorescent tracers exhibited movement parallel to the imaging plane. Known as transducer plane streaming ${ }^{39}$, this vortex-like flow has been previously observed in thin-reflector acoustofluidic devices, and is generated by the presence of both a standing and travelling wave components in the fluid cavity. The observation is backed by the modelling results presented in Section 0, where transducer-plane acoustic streaming patterns were revealed within the model fluid channel.

Within the vesicle lumen, a wide range of tracer flow patterns were observed (Figure 2 and 3). The average velocity of the beads was measured as $6.5 \pm 1.3 \mu \mathrm{m} / \mathrm{s}$, where the standard deviation represents the difference in average luminal velocity in the vesicle analysed in the channel. Figure 3 shows a representative image of the tracks generated by the PTV algorithm and the movement of an arbitrary particle translating within the vesicle lumen as a result of ultrasound exposure. It was also found that the luminal bead velocity was correlated with acoustic pressure within the fluid channel (Figure 1A). 

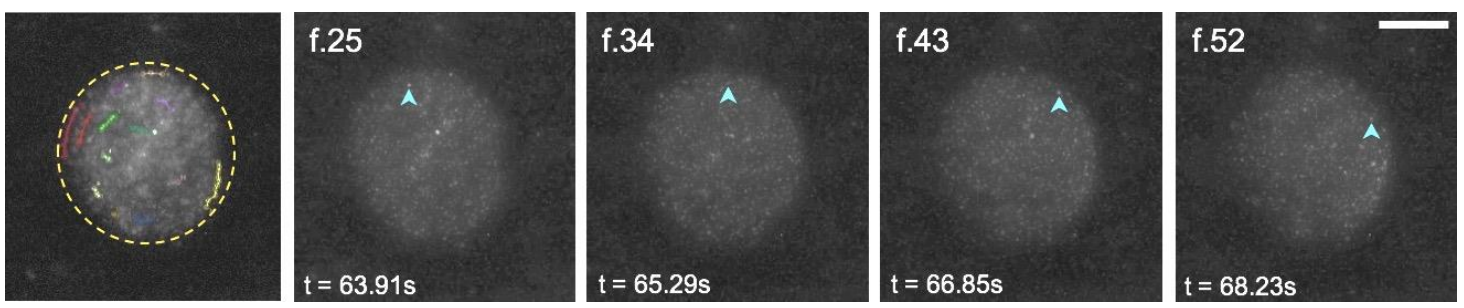

Figure 2 - Latex beads streaming within the lumen of a GUV. The cyan chevron indicates the position of a single bead in successive frames when exposed to $1 \mathrm{MHz}, 188 \mathrm{kPa}$ ultrasound in continuous wave. The frame number is denoted on the top left corner of each frame. Scale bar $=10 \mu \mathrm{m}$.

It was previously reported ${ }^{40,41}$ that in the presence of fluid flow external to the vesicles, the vesicle membrane behaves similarly to a fluid layer, allowing momentum to be transferred to the vesicle's lumen. This effect was observed within the acoustofluidic device and confirmed by the experiments presented in Figure 2. Numerical modelling also confirmed that ultrasound alone, in the absence of acoustic streaming, could induce luminal flows within the vesicles (see section 0.1).

\section{Ultrasound and microbubbles exposure}

When exposed to microbubbles and ultrasound, the average intravesicular velocity was recorded as $8.5 \pm 3.8 \mu \mathrm{m} / \mathrm{s}$ (Figure 1B). The flow patterns within the vesicle were heterogeneous and transient, as shown in Figure 3. This is likely due to the dynamic and stochastic nature of microbubble cavitation during ultrasound exposure. The primary and secondary radiation forces between microbubbles and beads coupled with the acoustic and cavitation microstreaming, lead to a larger average bead velocity compared to the case of ultrasound alone. The observed velocities were, however, still relatively modest and the effect of the microbubbles smaller than might be expected, given that the likelihood of ultrasound induced bioeffects is typically increased very significantly by the presence of microbubbles. A 
possible explanation is that vesicles in close proximity to cavitating microbubbles were in fact destroyed by the high shear stresses. Thus the velocities measured in the surviving vesicles were induced by relatively distant microbubbles and hence only slightly larger than those produced by ultrasound alone. It has been shown in previous work that cavitation microstreaming can generate much higher microstreaming velocities. ${ }^{42,43}$ Debris indicative of GUV destruction was observed in different areas of the microfluidic channel. In cholesteroldoped DOPC GUVs (5:1 molar ratio), Porter et al. reported a lysis tension of $1.89 \mathrm{mN} / \mathrm{m}^{44}$, and this may also be affected by the incorporation of microbubble DSPC debris onto the GUV surface $^{45}$, and/or by GUV-microbubble contact.

\section{Unidirectional flow}

Exposure to unidirectional flow was used as a control to determine whether flow alone was able to induce luminal streaming within the GUVs. Immediately upon activation of the flow, the fluorescent beads started to flow in two hemispherical patterns inside the vesicle, as predicted by the numerical simulations shown in Figure 5. The double vortex pattern is a result of the symmetrical forces imparted by the flow on the vesicle membrane. The average fluid velocity was determined to be $12 \pm 0.12 \mu \mathrm{m} / \mathrm{s}$, as shown in Figure $1 \mathrm{~B}$. The average fluid velocity did not show any correlation with the vesicle's diameter, as previously observed by Sebastien et al. ${ }^{40}$ While the vesicles at the centre of the channel exhibited symmetrical vortices, vesicles closer to the \pm y extremities exhibited non-symmetrical dipoles, with a smaller vortex on the side closer to the sidewall of the flow channel.

The results presented here are consistent with those reported in previous studies on phospholipid vesicles subjected to unidirectional flow. Membrane translation has been observed and quantified by Yamada et al. ${ }^{41}$, while intravesicle particle movement was first reported by Keller et al. ${ }^{46}$. Other studies have related the luminal flow velocity to the membrane properties of the vesicle's phospholipid membrane ${ }^{47}$. 


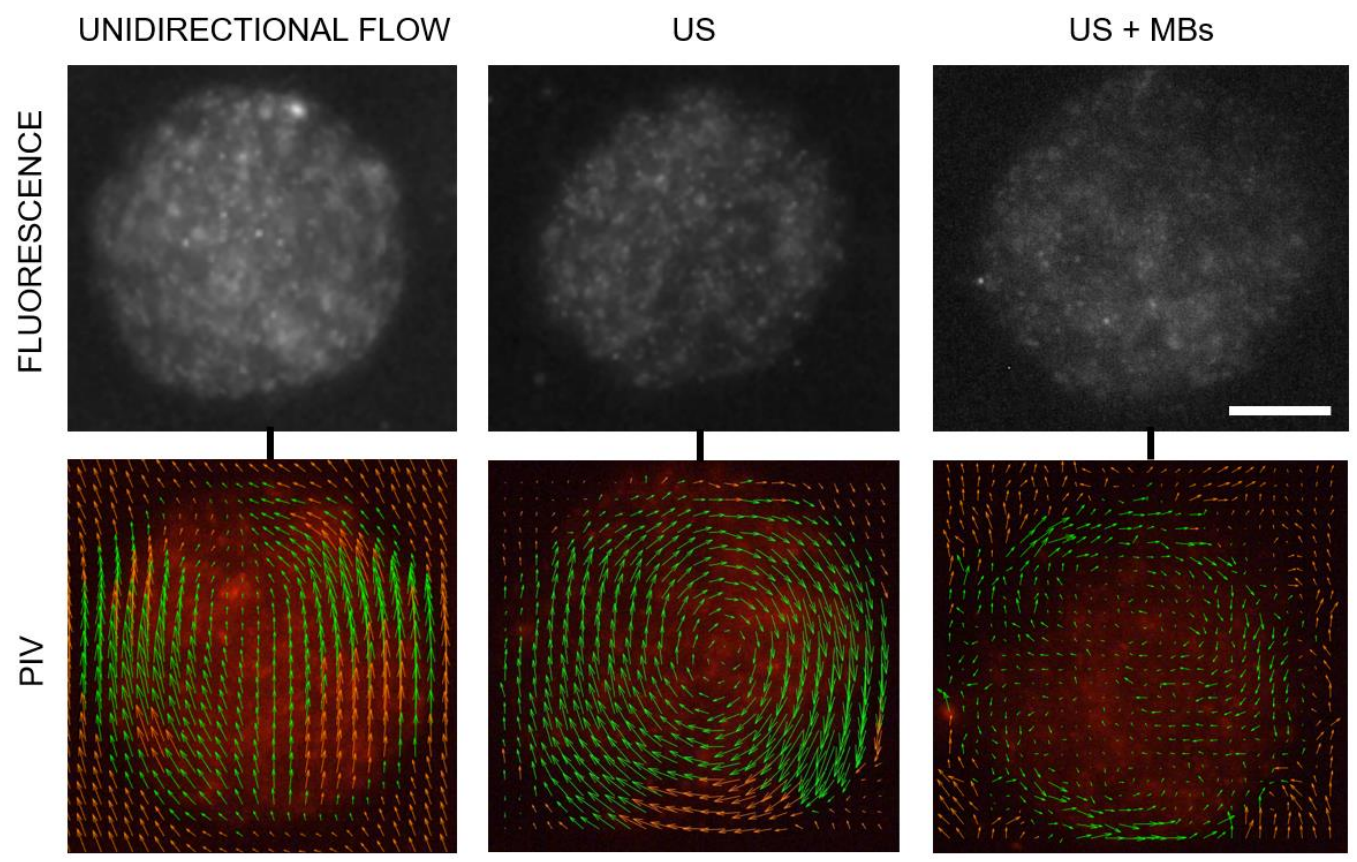

Figure 3 - (top) Fluorescence image frames of vesicles with encapsulated beads during varying exposure conditions. (bottom) Corresponding averaged flow fields generated using PIVLab®. Averaging was applied across 300 frames acquired during exposure. Interpolated vectors are labelled in orange. Vectors calculated throughout all frames, with at least six particle pairs, are labelled in green. Scale bar $=10 \mu \mathrm{m}$.

\section{Simulation results}

\section{Ultrasound exposure}

To determine whether ultrasound alone in the absence of external flow can generate streaming within the vesicle lumen, the acoustic streaming field inside the central vesicle was modelled using a slip condition at the membrane interface. The pressure field at the location of the vesicles was determined to be $160 \mathrm{kPa}$, consistent with the values recorded experimentally. When exposed to ultrasound, a well-formed transducer plane vortex, commonly reported in acoustofluidic devices, ${ }^{39,48}$ was observed within the model volume. This behavior was previously described by the authors ${ }^{48}$, and is a result of the combination of the SW and TW modes that lead to four-quadrant transducer-plane streaming patterns in the main fluid channel. 
Inside the vesicle, a rotational flow pattern was observed (Figure 4) despite the slip condition applied at the vesicle hemi-spherical boundary, which corresponded to the experimental observations presented in Figure 3. This shows that, even in the absence of outer flow, ultrasound causes streaming within the lumen of the vesicle. The maximum streaming velocity (i.e. the maximum limiting velocity at the bottom surface) in the modelled vesicle was found to be $11.36 \mu \mathrm{m} / \mathrm{s}$ (and an average velocity of $\sim 2.6 \mu \mathrm{m} / \mathrm{s}$ ), which is in line with the values obtained experimentally.
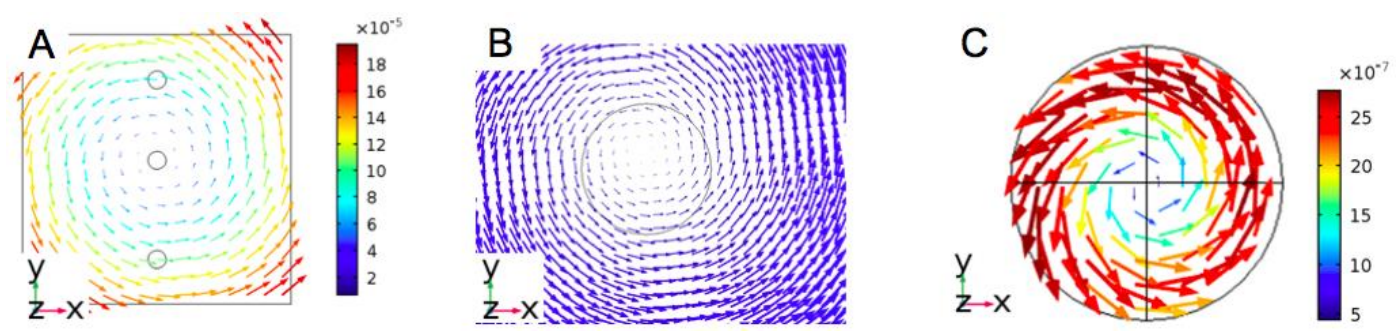

Figure 4 -A. the limiting velocity vector field at the bottom surface (the water-glass interface); B. the limiting velocity vector field inside and outside of the central vesicle at the bottom surface; and C. top view of the acoustic streaming velocity vector field in the central vesicle shown in A.

\section{Exposure to unidirectional flow}

When exposed to unidirectional flow, the modelled 3D flow fields inside the vesicles show two vortices in the $x y$ and $x z$ cross-sections. In the case of the central vesicle, these were specular, given the symmetric distribution of fluid flows on the vesicle in the $y$ direction of the fluid channel. For the vesicle closer to the channel edge, the vortexes were asymmetrical, with a smaller vortex on the side closer to the edge of the channel. Detailed flow fields at three $x y$ planes are plotted in Figure 5. 

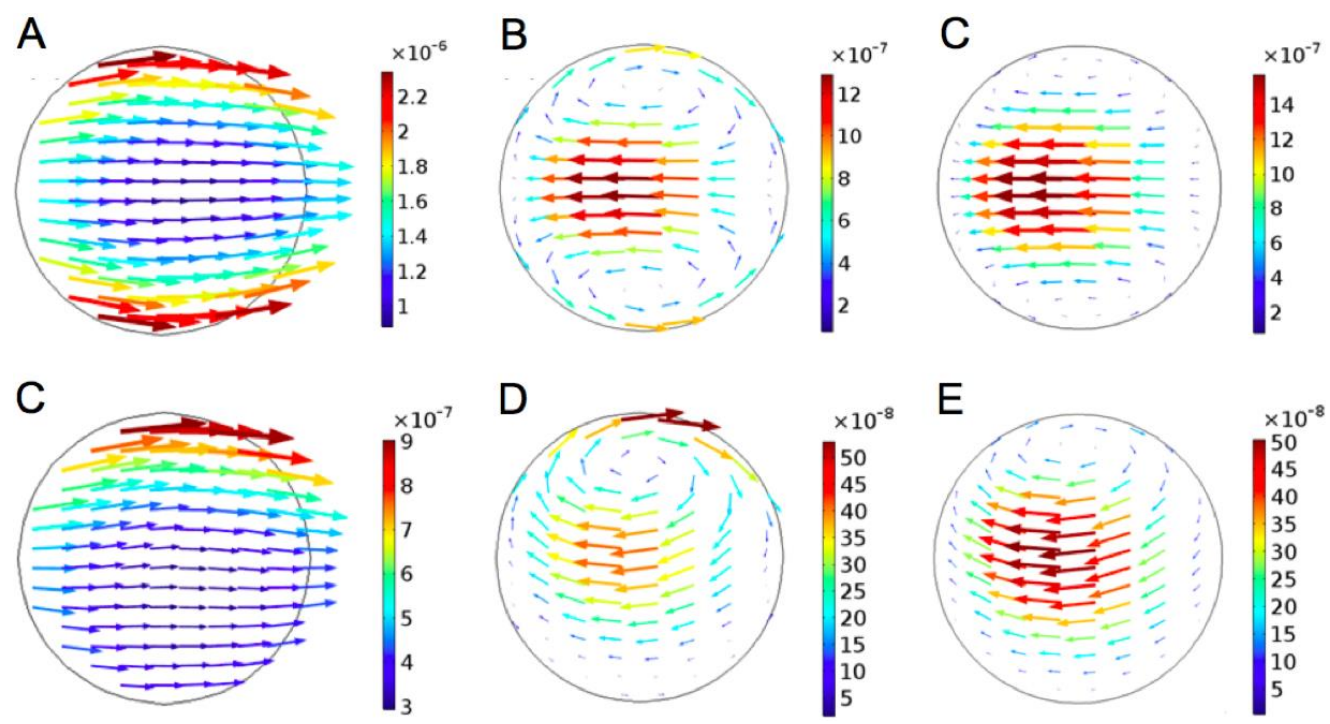

Figure 5 - (top) the detailed flow velocity vector fields (unit: $\mathrm{m} / \mathrm{s}$ ) on various $x y$ planes (measurement planes in experiments): (a) $z=-0.09 \mathrm{~mm}$; (b) $z=-0.096 \mathrm{~mm}$; (c) $z=$ $-0.099 \mathrm{~mm}$. (bottom) the detailed flow velocity vector fields (unit: $\mathrm{m} / \mathrm{s}$ ) on various $x y$ planes (measurement planes in experiments): (a) $z=-0.09 \mathrm{~mm}$; (b) $z=-0.096 \mathrm{~mm}$; (c) $z=$ $-0.099 \mathrm{~mm}$.

The flow patterns inside the central vesicle are shown in Figure 5A-C, while Figure 5C-E show the patterns for the vesicle closer to the $\pm y$ extremities of the fluid channel. Figure 5A shows the fluid patterns on the $x-y$ plane corresponding to $z=-0.09 \mathrm{~mm}$, which resembles the experimental observations reported by others ${ }^{40,41}$. The other $z$ cross-sections within the central vesicle (Figure 5B-C) reveal the 3D nature of the luminal flows, showing the varying position of the maximum velocity at different $z$-positions within the vesicle. Flow vortexes also formed for the vesicle closer to the edge of the channel, however these were not symmetrical (Figure 5C-E). Three-dimensional modelling of the flows for both cases are shown in Figures 1 and 2 in the Supporting Information. The results presented in this section confirm that flow, in the absence of ultrasound, also leads to luminal flow within vesicles, as shown previously experimentally. 


\section{Biological implications and limitations of the model}

The healthy functioning of mammalian cells relies on the active and passive transport of molecules between cellular compartments. For example, it has been shown that intra-cellular flows of cytosol play an important role in several cellular processes, including cell motility. ${ }^{49,50}$ Active transport velocities have been reported of the order of $0-3 \mu \mathrm{m} / \mathrm{s}^{25,51,52}$, which is well within the range of bead velocities reported in this study $(0.8 \pm 0.2 \mu \mathrm{m} / \mathrm{s}$ for no exposure, $12 \pm$ $0.12 \mu \mathrm{m} / \mathrm{s}$ for constant flow, $6.5 \pm 1.3 \mu \mathrm{m} / \mathrm{s}$ for ultrasound, $8.5 \pm 3.8 \mu \mathrm{m} / \mathrm{s}$ for ultrasound and microbubbles).

The fact that unidirectional flow produced significant internal streaming is also consistent with previous studies. Shear force transmission across lipid bilayers is one of the immediate effects of fluid flow on living cells and has been an area of research for many years. For example, it has been widely demonstrated that endothelial cells are sensitive to both magnitude and spatiotemporal gradients of wall shear stress ${ }^{53}$. The findings presented above suggest that ultrasound with and without microbubbles also has the potential to alter the spatial and temporal characteristics of shear force transmission across a lipid cell membrane, and this may have important implications. This study employed very mild ultrasound exposure conditions, such that the fluid velocities measured were in fact lower than those produced by unidirectional flow. Intravesicle streaming velocities would, however, be expected to increase with increasing ultrasound pressure (Figure 1B) and, as indicated above, vesicle destruction may have biased the velocity measurements towards lower values in the ultrasound and microbubble case.

Even allowing for the mild exposure conditions, the measured velocities, if generated in living cells would be of sufficient magnitude to produce significant biological effects. These could include alterations in ion balance, protein trafficking, and cargo transport, potentially leading to altered cellular functions and pathogenesis $;{ }^{54}$ or, alternatively, assisting therapeutic delivery 
by enabling transport of drug molecules to cellular compartments. Thus, in assessing the implications of the results, it is important to review how both the cell model and acoustofluidic device used in this study differ from living tissue.

In order to generate a uniform acoustic pressure field within the chamber and achieve therapeutically meaningful pressure magnitudes, the acoustofluidic device is constructed from materials of much greater rigidity than soft tissue. This would not be expected to affect the intra vesicle fluid motion generated by unidirectional flow. On the other hand, the deliberate minimization of a travelling wave component in the ultrasound field will have reduced the incidence of acoustic streaming ${ }^{48}$ and hence the effect of ultrasound alone. The presence of a rigid boundary will also affect microbubble dynamics, in particular the direction of microbubble translation and microjet formation. ${ }^{55,56}$ The impact on microstreaming and hence intra vesicle flow however, would be expected to be smaller, as the presence of any boundary will promote streaming. In fact a rigid boundary would be expected to reduce the amplitude of microbubble oscillation and hence streaming velocities compared to a more compliant one; ${ }^{.57}$ although the impact of tissue microstructure would also need to be taken into account.

With regard to the vesicles themselves, whilst their luminal volume is similar to that of cells, there are considerable differences with mammalian cells which could inhibit intracellular streaming, in particular: the poroviscoelastic nature of the cytoplasm and presence of the cytoskeleton. The vesicle membrane itself may also affect internal flow. Work by Sebastian et al. has shown that varying membrane composition by changing cholesterol content affected the efficiency of mechanotransmission and hence the luminal fluid velocity induced by external flow. ${ }^{59}$ Notwithstanding these limitations, the fact that ultrasound and microbubbles seem to be capable of producing intra vesicle flow velocities of the same order of magnitude as 
physiological flow would suggest that further investigation of potential bioeffects is warranted, both for understanding any safety implications and potential exploitation in therapy.

\section{Conclusions}

DOPC giant unilamellar vesicles doped with cholesterol were exposed to unidirectional flow, ultrasound, and ultrasound in combination with microbubbles. The resulting flows within the vesicle lumen were observed and quantified using particle tracking velocimetry. In the control cases, no particle movement was observed other than that due to Brownian motion. All other exposure conditions produced measurable particle movement. Upon exposure to ultrasound, luminal streaming patters were observed. Upon the introduction of microbubbles, the additional fluid motion created by bubble cavitation produced particle motion with variations in both magnitude and direction, suggestive of the effects of microbubble cavitation. Numerical modelling confirmed that exposure to ultrasound, even in the absence of flow outside the vesicles, can produce transducer-plane luminal acoustic streaming patterns with velocities analogous to those observed experimentally. Both modelling and experimental results showed that fluid flow in proximity of the vesicles is able to set up luminal streaming patterns, confirming results previously reported in the literature $40,60,61$. Intracellular perturbations and motion underpin a wide range of cellular functions such as ionic and osmotic regulation, the recruitment and transport of cargo, and reproduction. In this study we demonstrate that external flow, ultrasound and microbubbles, alone and in combination, all cause increased luminal fluid motion. The results presented in this study thus strongly motivate the need for further investigation of potential bioeffects caused by intracellular streaming. 


\section{Experimental Section}

Methods

Design and description of the acoustofluidic device

A previously developed, purpose-built thin-reflector acoustofluidic flow chamber $^{62}$ (i.e. a microfluidic chip with built-in acoustic field source) was employed for all experiments (Supporting Information Figure 3). The device consists of a piezoelectric transducer coupled using epoxy resin to a $1.2 \mathrm{~mm}$ MACOR $®$ ceramic carrier layer, a $0.2 \mathrm{~mm}$ thick fluid channel, and a $0.17 \mathrm{~mm}$ thick glass reflector. Layers are held together by a metal frame and a Perspex ${ }^{\circledR}$ manifold. The acoustofluidic device was designed to allow ultrasound exposure at a therapeutically relevant frequency of $1 \mathrm{MHz}$, and acoustic pressures of up to $400 \mathrm{kPa}$. The device was also intended to function as a flow cell to enable the exposure of the vesicles to fluid flow, imparting a relatively uniform wall shear stress field.

\section{Formation of vesicles with encapsulated flow tracers}

Giant vesicles were produced on stainless steel needles using a previously described electroformation protocol $^{63}$. In brief, commercially available injection needles (Becton Dickinson, UK) were first cleaned with toluene (Fisher Scientific, UK) and left to dry overnight. A chloroform lipid solution (Avanti Polar Lipids, USA) composed of DOPC and cholesterol (Sigma Aldrich, UK) was prepared at a molar ratio of 3:1. 1,2-dipalmitoyl-snglycero-3-phosphoethanolamine-N-(cap biotinyl) (sodium salt) was then added to the solution at $1 \mathrm{~mol} \%$ to biotinylate the lipids, allowing the subsequent fixation of the vesicles on the bottom surface of the acoustofluidic device. The solution was then mixed thoroughly and carefully pipetted on the outer surface of the needles using a glass syringe. After a drying phase of approximately one hour, the needle pair was assembled $3 \mathrm{~mm}$ apart, and submerged in a 
solution of water and sucrose $(200 \mathrm{mM})$ containing monodisperse $500 \mathrm{~nm}$ (in diameter) carboxylate fluorescent latex beads (Sigma Aldrich, UK). The size of the monodisperse beads was chosen to be significantly smaller than the hydrodynamic length scale of the channel, i.e. the channel height, in order to obtain a suitable compromise between visibility and flow fidelity. ${ }^{64}$ The ratio between fluid and particle density, and kinematic viscosity, resulted in a particle response time orders of magnitudes smaller than the smallest time scale of the flow $(\mu \mathrm{m} / \mathrm{s})$. In addition, the primary acoustic radiation forces on sub-micrometre particles would be expected to be small, as these scale with particle volume. A sinusoidal voltage with a peakto-peak amplitude of $4.86 \mathrm{~V}$ and a frequency of $10 \mathrm{~Hz}$ was then applied to the electrodes for two hours. The frequency was subsequently lowered to $5 \mathrm{~Hz}$ to stimulate vesicle detachment from the electrode surface. The needles were removed, and an aliquot of the resulting beadloaded vesicle suspension was mixed with an equal amount of PBS, to promote vesicle sedimentation upon injection into the acoustofluidic device. The average size of the vesicles was determined optically using the proprietary Zeiss Zen Black software. Figure 6B shows a colourised confocal image of three vesicles containing fluorescent beads. The average diameter of the vesicles was measured to be $31 \pm 17 \mu \mathrm{m}$. Whilst the diameter of the vesicles is larger than that of typical live cells $(\sim 20 \mu \mathrm{m})$, simulations indicated that this would not be expected to have a significant impact on the results.

\section{Fixation of vesicles onto the glass coverslip}

To provide a more realistic model of adherent cells, vesicles were bound to the glass reflector layer using an avidin-biotin linkage. This was achieved by doping the vesicles with biotinylated lipids prior to the formation process (as described in Section 2.1.2), and by coating the glass slide with biotinylated serum albumin (bBSA) and subsequently with avidin from egg white, as described previously by Kuhn et al. ${ }^{65}$ Upon priming the chamber with the vesicle-rich 
solution, vesicles sediment due to the density difference between sucrose and PBS (1.02 and $1.00394^{66} \mathrm{~g} / \mathrm{cm}^{3}$, respectively) and, coming into contact with the glass slide, bind with the avidin layer. After an incubation phase of approximately 10 minutes at room temperature, the device was flushed to remove excess latex beads. Figure 6A shows bound vesicles stained with a fluorescent lipid-analogue dye (c-Laurdan).

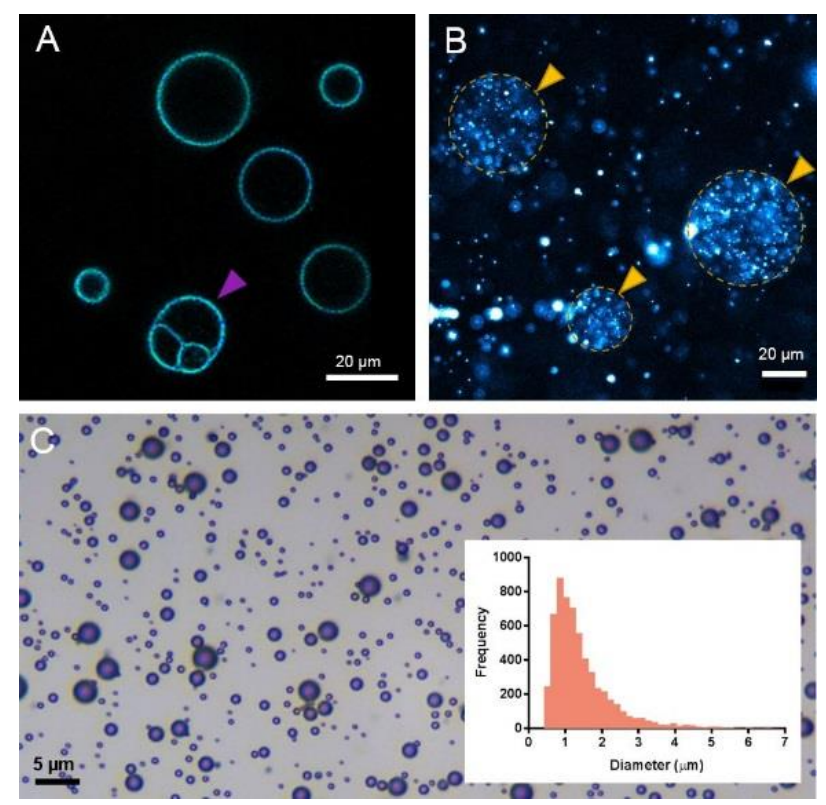

Figure 6 - A. Fluorescence confocal image of DOPC GUVs stained with c-Laurdan, imaged with a Zeiss plan apochromat $63 x$ objective mounted on a Zeiss 780 confocal microscope. The purple arrow indicates a representative multilamellar GUV that was excluded from the analysis. B. Confocal microscope image of three GUVs of different sizes with encapsulated fluorescent latex beads (500 $\mathrm{nm}$ in diameter) within their lumen, and bound to the glass surface. The image was acquired using a Zeiss LSM 780 confocal microscope equipped with a $63 x$ planApochromat oil-immersion objective. C. Suspension of DSPC:PEG40s 9:1 microbubbles imaged in a Neubauer haemocytometer for microbubble counting and sizing. Inset indicates microbubble size distribution of a representative sample. The images were acquired using a Nikon Ti Eclipse ${ }^{\circledR}$ fluorescence microscope with a 40x objective. Mean microbubble diameter $=1.47 \mu \mathrm{m} \pm 0.89 \mu \mathrm{m}$. 


\section{Formation and characterisation of microbubbles}

Lipid-shelled microbubble suspensions were formed as previously described by Carugo et al. ${ }^{17}$ by dissolving 1,2-distearoyl-sn-glycero-3-phosphocholine and polyethylene glycol 40 stearate in chloroform at a 9:1 molar ratio, respectively. It has been shown previously that there is a negligible difference between the response of commercially available SonoVue and these microbubbles under the range of ultrasound exposure conditions used in this study ${ }^{62}$.The resulting solution was then pipetted in a $15 \mathrm{~mL}$ glass vial and left to stand overnight on a hotplate at $50^{\circ} \mathrm{C}$ to allow the chloroform to evaporate completely. The vial was then filled with $5 \mathrm{~mL}$ of PBS and stirred for 30 minutes on a hotplate set to $100^{\circ} \mathrm{C}$. The solution was then sonicated for 150s with a tip sonicator (XL2000, 3mm, 20W, $22.5 \mathrm{kHz}$, Misonix, USA) fully submerged in the vial to uniformly disperse the lipids. A further sonication step was performed by submerging the sonicator tip (power setting 14) approximately $10 \mathrm{~mm}$ from the air-water interface for 20 seconds while blowing a stream of sulphur hexafluoride (The BOC Group, UK) at the interface. The vial was then placed in ice for 15 minutes and the suspension was washed three times by centrifugation (300 RCF, $10 \mathrm{~min}$ ) and diluted in PBS to reach a final concentration of $\sim 1 \times 10^{6}$ microbubbles $/ \mathrm{ml}$. The microbubble concentration calculation was performed, as shown in Figure 6C, using an algorithm developed by Sennoga et al. ${ }^{67}$

Exposure conditions

No exposure

Vesicles were imaged using an inverted Nikon Ti Eclipse ${ }^{\circledR}$ microscope equipped with a coolLED excitation system, at 40x magnification. Vesicles containing beads were imaged in fluorescence mode (excitation: $575 \mathrm{~nm}$, emission: $610 \mathrm{~nm}$ ), at 5 frames per second with an 
exposure time of $200 \mathrm{~ms}$ and 2x digital gain. To record a baseline bead velocity, vesicles were imaged continuously for one minute with no excitation. Only vesicles that were unilamellar and spherical were selected for all investigations reported in this manuscript. All experiments were performed at room temperature.

\section{Unidirectional flow}

A laminar Poiseuille flow was established in the fluid channel of the device using a syringe pump (Cole Palmer, UK) and a $20 \mathrm{ml}$ plastic syringe (Becton Dickinson, UK), to achieve an average wall shear stress of $1.25 \mathrm{~Pa}$ over the inner glass surface of the fluid channel. Physiological flow velocities range from $\sim 30 \mu \mathrm{m} / \mathrm{s}$ in capillaries, $15 \mathrm{~mm} / \mathrm{s}$ in the vena cava, and $40 \mathrm{~cm} / \mathrm{s}$ in the aorta. Physiological shear stress levels range from 0.1 to $6 \mathrm{~Pa}$ in the venous system with peaks of $7 \mathrm{~Pa}$ in arteries ${ }^{68}$. The flow rate was chosen as an approximate value of the cavitation microstreaming flows (in the order of a few $\mathrm{mm} / \mathrm{s}$ ) previously recorded in the same device by the authors. ${ }^{9}$

\section{Ultrasound exposure}

The piezoelectric chip was driven with a $39.4 \mathrm{~V}$ sinusoidal input with a frequency of 1.014 MHz, generated using an Agilent 33220A arbitrary waveform generator followed by $55 \mathrm{~dB}$ amplification using a RF amplifier (E\&I, USA). The resulting peak negative acoustic pressure in the fluid channel was quantified by inserting a fibre-optic hydrophone within the fluid layer corresponding to the position of the piezoelectric chip, and was determined to be $188 \mathrm{kPa}$, which is within the therapeutic range used in microbubble-based ultrasound mediated therapy ${ }^{69-71}$. Vesicles were exposed to ultrasound for one minute continuously.

\section{Ultrasound and microbubble exposure}

Vesicles were exposed to ultrasound in the presence of microbubbles for one minute. This was 
achieved by injecting microbubbles at a final concentration of $1 \times 10^{6} \mathrm{MB} / \mathrm{ml}$ into the fluid channel, and immediately activating the ultrasound field with the same parameters as described in section 2.1.5.3.

\section{Bead velocity analysis}

Tracking of single fluorescent beads was performed using the native ImageJ® TrackMate ${ }^{\circledR}$ plugin. The dynamics of single beads was monitored on a frame-by-frame basis to reveal individual particle trajectories and velocities. First, a region of interest was determined and cropped to isolate a single GUV. Secondly, beads were detected automatically using the builtin detection algorithm, and were individually identified on each frame pair. Thirdly, the average bead velocity was determined by averaging the velocities in each frame pair for all the bead trajectories detected. The individual trajectories were inspected manually to avoid detection errors that arise when two beads in two separate tracks overlap. The illustrative average flows in Figure 3 were generated using a time-resolved particle image velocimetry tool (PIVLab®) to obtain a representative flow profile for each exposure condition. Particle velocities were quantified bi-dimensionally, in the $x-y$ plane. Images were pre-processed to exclude stationary features in the field of view, subjected to a high pass filter with a $15 \times 15$ pixel area to improve particle detection, and calibrated using the width of the field of view (to convert pixels to physical units).

\section{Statistical analysis}

Statistical significance was determined by first performing a one-way ANOVA on all exposure conditions, followed by Tukey's multiple comparisons test between each condition and the respective control. 
Modelling of microstreaming flows within vesicles

This section describes the numerical models and methods employed to simulate flow patterns inside the vesicle lumen when exposed to ultrasound and unidirectional flow. The primary purpose of modelling the flow inside the vesicles is to determine whether ultrasound alone, in the absence of pressure-driven flow outside the vesicle membrane, can induce a flow within the vesicle lumen. The luminal flow as a result of external unidirectional flow in the acoustofluidic device was also investigated in order to determine whether the acoustic streaming and cavitation streaming alone may be contributing factors to the flow inside the vesicle lumen. A detailed description of the theory of acoustic streaming is included in the Supporting Information.

Microstreaming flows as a result of ultrasound exposure

\section{Numerical model}

A simplified 3D reduced-fluid model with dimensions of $0.3 \times 0.3 \times 0.2 \mathrm{~mm}^{3}$ was considered (Figure 7A) in COMSOL to mimic a portion of the experimental fluid channel with dimensions $60 \times 12 \times 0.2 \mathrm{~mm}$. Surfaces $\mathrm{z}=0.1$ and $\mathrm{z}=-0.1 \mathrm{~mm}$ represent the carrier-fluid and fluidreflector interfaces respectively. Three hemispheres with lateral positions $y=0$ and $y= \pm 100$ $\mu \mathrm{m}$ were used to represent the vesicles adherent to the glass surface of the chamber. Three vesicles were modelled to determine the luminal flow patterns in the presence and absence of external flow. The central vesicle was placed in correspondence with the acoustic pressure node in the y-direction and the centre of the acoustic streaming vortex, in order to investigate the effect of ultrasound on intravesicle flows in the absence of microstreaming. For mesh constitutions, as shown in Figure 7B, uniform tetrahedral mesh was used in the bulk of the fluid 
channel while smaller elements were used in vesicles to resolve the acoustic and streaming fields near the curved surfaces of the vesicles

A

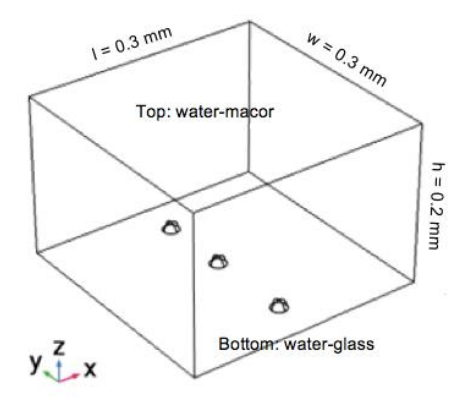

B

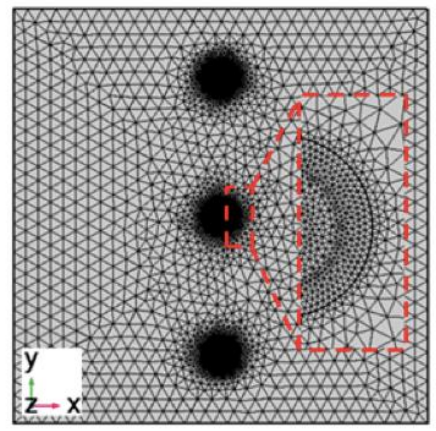

C

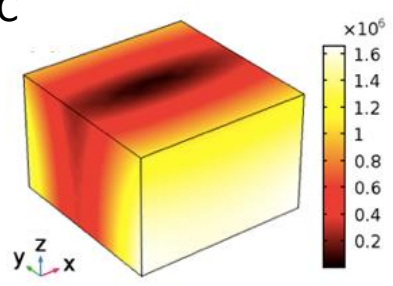

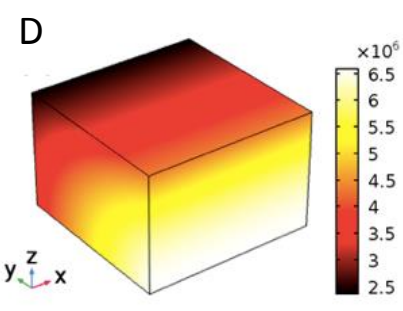

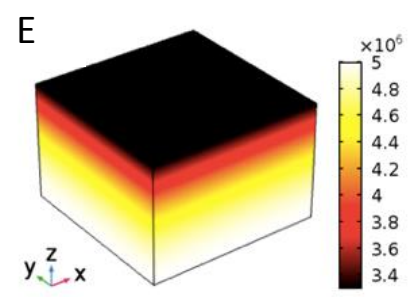

Figure 7 - A. the 3D model considered; B. mesh condition at the bottom surface of the model (the fluid-reflector interface). C. the total 3D acoustic pressure magnitudes in the model regime $(\mathrm{Pa})$; D. the standing wave component $(\mathrm{Pa})$; E. the travelling wave component $(\mathrm{Pa})$.

\section{Definition of the acoustic field}

The linear acoustic fields in the whole fluid channel were modelled using a 'Pressure Acoustics, Frequency Domain' interface, which solves the harmonic linearized acoustics, taking the form:

$$
\nabla^{2} p_{1}+\frac{\omega^{2}}{c^{2}} p_{1}=0
$$

where $\omega$ is the angular frequency and $c$ is the speed of sound in the fluid. The acoustic fields in the model regime were created by harmonic vibrations of the upper surface and walls of the 
device coupled with reflecting boundary conditions at each of the inner surfaces. The vibration of the upper surface creates the main thickness standing wave field in the fluid cavity of the acoustofluidic device. Acoustic pressure gradients in the lateral directions caused by harmonic vibrations of the walls were considered in order to account for energy gradients in directions other than the main direction of ultrasound propagation (which are commonly observed in thinreflector acoustofluidic devices ${ }^{39,72}$ ).

To do this, in addition to the linear acoustic model used to approximate the $3 \mathrm{D}$ first-order acoustic field in the channel, an additional travelling wave component propagating along the fluid channel $\left(\mathrm{p}_{1 \mathrm{t}}\right)$ in the $\mathrm{x}$-direction was considered. Their phase difference has been shown to create transducer plane streaming (TPS) in acoustofluidic devices of similar geometry. The acoustic field in the fluid channel may be represented as a summation of the standing wave (SW) field and the travelling wave (TW) component:

$$
\begin{gathered}
p_{1}=p_{1 s}+p_{1 t}, \\
p_{1 s}=p_{0 s} \cos \left(k_{x s} x\right) \cos \left(k_{y s} y\right) \sin \left(k_{z s} z\right) e^{i \omega t}, \\
p_{1 t}=p_{0 t} e^{i k_{x t} x} \cos \left(k_{y t} y\right) \sin \left(k_{z t} z\right) e^{i(\omega t+\varphi)},
\end{gathered}
$$

where the second subscripts $s$ and $t$ indicate the SW and TW components respectively, $\mathrm{p}_{0}$ is the acoustic pressure amplitude, $\omega$ is the angular frequency, $\mathrm{k}_{\mathrm{x}}, \mathrm{k}_{\mathrm{y}}$ and $\mathrm{k}_{\mathrm{z}}$ are the wave numbers in the $\mathrm{x}, \mathrm{y}$ and $\mathrm{z}$ directions and $\varphi$ indicates the phase difference between the $\mathrm{SW}$ and TW components.

The total acoustic pressure field was defined as a combination of a $(1,2,1) \mathrm{SW}$ mode and a ( $\mathrm{t}$, $0,1)$ TW mode in a fluid channel with dimensions of $60 \times 12 \times 0.74 \mathrm{~mm}^{3}$, which results in a resonant frequency of $1.0077 \mathrm{MHz}$, which is comparable to the experimentally verified 
frequency. The pressure amplitude in the model was approximately $0.16 \mathrm{MPa}$, also similar to the value measured experimentally. Diagrams of the acoustic pressure inside the simplified 3D fluid model are shown in Figure 7C-E.

\section{Definition of acoustic streaming fields}

The limiting velocity method ${ }^{73,74}$, which is based on the perturbation method ${ }^{75}$, was used to generate the 3D acoustic streaming fields in the acoustofluidic device and inside the vesicles. The acoustic streaming fields within the vesicles were modelled using the 'Creeping Flow' interface, assuming the 3D flow fields in the vesicles are fully generated from the acoustic streaming fields just outside the vesicle membranes. To determine the effect of ultrasound alone in the absence of external flow, a slip velocity boundary condition on the hemi-spherical surface of the vesicles was applied, with the exception of the bottom surface, where a no-slip condition was considered.

In the model, the overall streaming field is dominated by boundary-driven streaming, while Eckart streaming is considered to be negligible given the small length scales $(<200 \mu \mathrm{m})$. As a result, only the boundary-driven streaming was modelled. As the inner streaming vortices are confined to the viscous boundary layer only (thickness of $\delta_{\mathrm{v}} \approx 0.6 \mu \mathrm{m}$ at $1 \mathrm{MHz}$ in water ${ }^{76}$ ), for numerical efficiency, only the 3D outer streaming fields were solved using Nyborg's limiting velocity method ${ }^{73,74}$, as performed by previous studies ${ }^{39,48,75,77,78}$, and evaluated by Lei et al. $^{79}$

\section{Derivation of limiting velocity fields}


The limiting velocities at the driving boundaries were calculated as a function of the first-order acoustic velocity fields. On planar surfaces normal to $\mathrm{z}$, the limiting velocity equations on the driving boundaries take the form:

$$
\begin{gathered}
u_{L}=-\frac{1}{4 \omega} \operatorname{Re}\left\{q_{x}+u_{1}^{*}\left[(2+i) \nabla \cdot \boldsymbol{u}_{\mathbf{1}}-(2+3 i) \frac{d w_{1}}{d z}\right]\right\}, \\
v_{L}=-\frac{1}{4 \omega} \operatorname{Re}\left\{q_{y}+v_{1}^{*}\left[(2+i) \nabla \cdot \boldsymbol{u}_{1}-(2+3 i) \frac{d w_{1}}{d z}\right]\right\}, \\
q_{x}=u_{1} \frac{d u_{1}^{*}}{d x}+v_{1} \frac{d u_{1}^{*}}{d y}, \\
q_{y}=u_{1} \frac{d v_{1}^{*}}{d x}+v_{1} \frac{d v_{1}^{*}}{d y},
\end{gathered}
$$

where $\mathrm{u}_{\mathrm{L}}$ and $\mathrm{v}_{\mathrm{L}}$ are the two components of limiting velocities along coordinates $\mathrm{x}$ and $\mathrm{y}, \operatorname{Re}$ represents the real part of a complex value, and $\mathrm{u}_{1}, \mathrm{v}_{1}$ and $\mathrm{w}_{1}$ are components of the complex first-order acoustic velocity vector, $\mathrm{u}_{1}$, along the coordinates $\mathrm{x}, \mathrm{y}$ and $\mathrm{z}$, respectively.

\section{Modelling of acoustic streaming fields}

The limiting velocities at the driving boundaries were calculated as a function of the first-order acoustic velocity fields as shown:

$$
\begin{gathered}
\nabla \mathrm{p}_{2}=\mu \nabla^{2} \mathrm{u}_{2} \\
\nabla \cdot \mathrm{u}_{2}=0
\end{gathered}
$$

where $\mu$ is the dynamic viscosity of the fluid. The bottom surface of the vesicle was considered as a limiting velocity boundary condition and the remaining surfaces as no-slip boundary conditions. 
Flow within vesicles as a result of unidirectional flow

\section{Model description}

To study the flow within the vesicles resulting from unidirectional flow inside the acoustofluidic channel, a three-dimensional (3D) fluidic model was considered, as shown in Figure 8A. The model has a dimension of $0.3 \times 0.3 \times 0.2 \mathrm{~mm}^{3}(x \times y \times z)$. The coordinates $x$, $y$ and $z$ are the continuous flow direction, lateral direction and thickness direction, respectively. Surfaces $z=0.1$ and $z=-0.1 \mathrm{~mm}$ represent respectively the carrier-fluid and fluid-reflector interfaces. Two hemispheres on the bottom glass surface, with lateral positions of $y=0$ and $y=135 \mu \mathrm{m}$, were used as cell models to study the flows in the vesicles at the central region (i.e. $y=0$ ) and near side boundaries (i.e. $y= \pm w / 2$ ) as shown in Figure 8A.

A continuous laminar flow developed in the $+x$ direction was considered, shown in Figure 8B, where the flow field on a $y z$ cut-plane through the vesicles is plotted. The flow velocity is maximum at the centre of the channel and zero at boundaries.

A

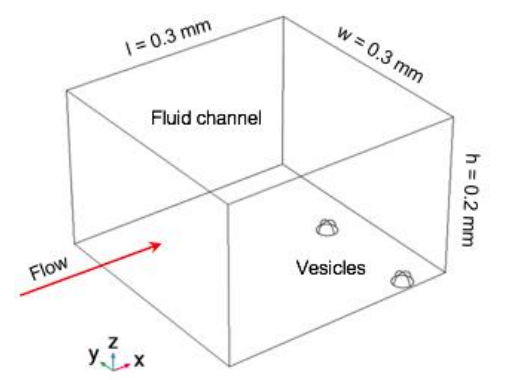

B

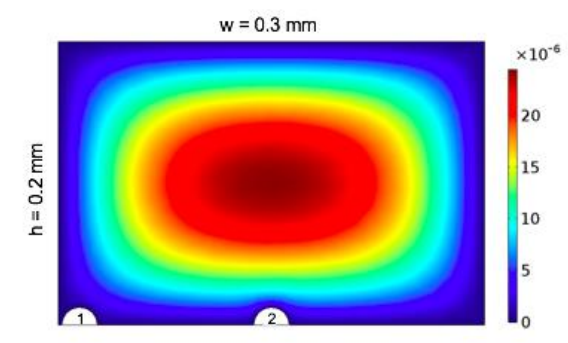

Figure 8 - A. the three-dimensional model considered, where two vesicles are located at the bottom surface $(y=0$ and $y=135 \mathrm{~mm}$ ); and $\mathrm{B}$. the contour of fluid velocity magnitude (unit: $\mathrm{m} / \mathrm{s}$ ) in the $y z$ cross-section of the fluid channel. $z=0.1$ and $z=-0.1 \mathrm{~mm}$ are the watermacor and water-glass interfaces, respectively. The radius of vesicle is $r=12.5 \mu \mathrm{m}$. 


\section{Acknowledgements}

The authors would like to extend their gratitude to James Fisk and David Salisbury for the fabrication of the electroformation chamber and acoustofluidic device. The project was supported financially by The Engineering and Physical Sciences Research Council (grants EP/I021795/1 and EP/L024012/1) and the Institute of Engineering and Technology (AF Harvey Prize).

\section{Author contributions}

V.P. devised and conducted the experiments, J.L. devised and performed all numerical simulations, E.S and D.C. conceptualised and supervised the work. All authors reviewed the manuscript.

\section{Additional information}

Supporting information accompanies this paper at http://doi.org/XXX

Raw data are available through the University of Oxford ORA data repository DOI: XXXX

Competing interests: The authors declare that they have no competing interests. 


\section{References}

(1) Stride, E. P.; Coussios, C. C. Cavitation and Contrast: The Use of Bubbles in Ultrasound Imaging and Therapy. Proc. Inst. Mech. Eng. Part H J. Eng. Med. 2010, 224 (2). https://doi.org/10.1243/09544119JEIM622.

(2) Lentacker, I.; De Cock, I.; Deckers, R.; De Smedt, S. C.; Moonen, C. T. W. Understanding Ultrasound Induced Sonoporation: Definitions and Underlying Mechanisms. Advanced Drug Delivery Reviews. 2014. https://doi.org/10.1016/j.addr.2013.11.008.

(3) Collis, J.; Manasseh, R.; Liovic, P.; Tho, P.; Ooi, A.; Petkovic-Duran, K.; Zhu, Y. Cavitation Microstreaming and Stress Fields Created by Microbubbles. Ultrasonics 2010, 50 (2), 273-279. https://doi.org/10.1016/j.ultras.2009.10.002.

(4) Hu, Y.; Wan, J. M. F.; Yu, A. C. H. Membrane Perforation and Recovery Dynamics in Microbubble-Mediated Sonoporation. Ultrasound Med. Biol. 2013, 39 (12), 23932405. https://doi.org/10.1016/j.ultrasmedbio.2013.08.003.

(5) Chen, X.; Leow, R. S.; Hu, Y.; Wan, J. M. F.; Yu, A. C. H. Single-Site Sonoporation Disrupts Actin Cytoskeleton Organization. J. R. Soc. Interface 2014, 11 (95), 20140071-20140071. https://doi.org/10.1098/rsif.2014.0071.

(6) Prentice, P.; Cuschieri, A.; Dholakia, K.; Prausnitz, M.; Campbell, P. Membrane Disruption by Optically Controlled Microbubble Cavitation. Nat. Phys. 2005, 1 (2), 107-110. https://doi.org/10.1038/nphys148.

(7) van Wamel, A.; Kooiman, K.; Harteveld, M.; Emmer, M.; ten Cate, F. J.; Versluis, M.; de Jong, N. Vibrating Microbubbles Poking Individual Cells: Drug Transfer into Cells via Sonoporation. J. Control. Release 2006, 112 (2), 149-155. https://doi.org/10.1016/j.jconrel.2006.02.007. 
(8) Wu, J. Shear Stress in Cells Generated by Ultrasound. Prog. Biophys. Mol. Biol. 2007, 93 (1-3), 363-373. https://doi.org/10.1016/j.pbiomolbio.2006.07.016.

(9) Valerio, P.; Miles, A.; Oliver, V.; Mannaris, C.; Anjali, S.; Victor, de Saint;

Guillaume, L.; Michel, V.; Dario, Coussios Constantin Carugo; stride, E. Layered Acoustofluidic Resonators for the Generation of Cavitation, Microstreamie, Oliver; Christophoros Mannaris; Seth, Anjali; de Saint Victor; Lajoinie, Guillaume; Versluis, Michel; Coussios, Constantin, Carugo, Dario; Stride, Eleanor;Ng, and Biologica. Unpublished.

(10) Prosperetti, A.; Crum, L. A.; Commander, K. W. Nonlinear Bubble Dynamics. J. Acoust. Soc. Am. 1988, 83 (2), 502-514. https://doi.org/10.1121/1.396145.

(11) Wu, J.; Ross, J. P.; Chiu, J.-F. Reparable Sonoporation Generated by Microstreaming. J. Acoust. Soc. Am. 2002, 111 (3), 1460-1464. https://doi.org/10.1121/1.1420389.

(12) Yu, H.; Xu, L. Cell Experimental Studies on Sonoporation: State of the Art and Remaining Problems. J. Control. Release 2014, 174 (1), 151-160. https://doi.org/10.1016/j.jconrel.2013.11.010.

(13) Zhong, W.; Sit, W. H.; Wan, J. M. F.; Yu, A. C. H. Sonoporation Induces Apoptosis and Cell Cycle Arrest in Human Promyelocytic Leukemia Cells. Ultrasound Med. $\{\&\}$ Biol. 2011, 37 (12), 2149-2159. https://doi.org/10.1016/j.ultrasmedbio.2011.09.012.

(14) Haidekker, M. A.; L’Heureux, N.; Frangos, J. A. Fluid Shear Stress Increases Membrane Fluidity in Endothelial Cells: A Study with DCVJ Fluorescence. Am. J. Physiol. Heart Circ. Physiol. 2000, 278 (4), 1401-1406.

(15) Yamamoto, K.; Ando, J. Vascular Endothelial Cell Membranes Differentiate between Stretch and Shear Stress through Transitions in Their Lipid Phases. Am. J. Physiol. Heart Circ. Physiol. 2015, 309 (7), ajpheart.00241.2015. https://doi.org/10.1152/ajpheart.00241.2015. 
(16) Yamamoto, K.; Ando, J. Endothelial Cell and Model Membranes Respond to Shear Stress by Rapidly Decreasing the Order of Their Lipid Phases. J. Cell Sci. 2013, 126 (5), 1227-1234. https://doi.org/10.1242/jcs.119628.

(17) Carugo, D.; Aron, M.; Sezgin, E.; Bernardino de la Serna, J.; Kuimova, M. K.; Eggeling, C.; Stride, E. Modulation of the Molecular Arrangement in Artificial and Biological Membranes by Phospholipid-Shelled Microbubbles. Biomaterials 2017, 113, 105-117. https://doi.org/10.1016/j.biomaterials.2016.10.034.

(18) Šlotová, J.; Kaepfel, Z.; Hrazdira, I. Chromosome Aberrations Caused by the Effect of Ultrasound in the Meristematic Cells Ofvicia Faba. Biol. Plant. 1967, 9 (1), 49-55. https://doi.org/10.1007/BF02930735.

(19) Miller, M. W.; Ciaravino, V.; Allen, D.; Jensen, S. Effect of 2 MHz Ultrasound on DNA, RNA and Protein Synthesis in Pisum Sativum Root Meristem Cells. Int. J. Radiat. Biol. 1976, 30 (3), 217-222. https://doi.org/10.1080/09553007614550991.

(20) Miller, D. L. The Botanical Effects of Ultrasound: A Review. Environ. Exp. Bot. 1983, 23 (1), 1-27. https://doi.org/10.1016/0098-8472(83)90017-5.

(21) Miller, D. L. A Review of the Ultrasonic Bioeffects of Microsonation, Gas-Body Activation, and Related Cavitation-like Phenomena. Ultrasound Med. Biol. 1987, 13 (8), 443-470. https://doi.org/10.1016/0301-5629(87)90110-4.

(22) Hussein, F.; Antonescu, C.; Karshafian, R. Ultrasound and Microbubble Induced Release from Intracellular Compartments. BMC Biotechnol. 2017, 17 (1), 1-12. https://doi.org/10.1186/s12896-017-0364-3.

(23) Yang, F.; Gu, N.; Chen, D.; Xi, X.; Zhang, D.; Li, Y.; Wu, J. Experimental Study on Cell Self-Sealing during Sonoporation. J. Control. Release 2008, 131 (3), 205-210. https://doi.org/10.1016/j.jconrel.2008.07.038.

(24) Mateus, A.; Treyer, A.; Wegler, C.; Karlgren, M.; Matsson, P.; Artursson, P. 
Intracellular Drug Bioavailability: A New Predictor of System Dependent Drug Disposition. Sci. Rep. 2017, 7 (1), 43047. https://doi.org/10.1038/srep43047.

(25) McPherson, P. S.; Ritter, B.; Wendland, B. Trafficking Inside Cells: Pathways, Mechanisms and Regulation; Landes Bioscience, 2009; Vol. 1. https://doi.org/10.1007/978-0-387-93877-6_7.

(26) Schlicher, R. K.; Radhakrishna, H.; Tolentino, T. P.; Apkarian, R. P.; Zarnitsyn, V.; Prausnitz, M. R. Mechanism of Intracellular Delivery by Acoustic Cavitation. Ultrasound Med. Biol. 2006, 32 (6), 915-924. https://doi.org/10.1016/j.ultrasmedbio.2006.02.1416

(27) Tang, Y.; Olufemi, L.; Wang, M.-T.; Nie, D. Role of Rho GTPases in Breast Cancer. Front. Biosci. 2008, 13 (13), 759-776. https://doi.org/10.2741/2718.

(28) Dugani, C. B.; Klip, A. Glucose Transporter 4: Cycling, Compartments and Controversies. EMBO Rep. 2005, 6 (12), 1137-1142. https://doi.org/10.1038/sj.embor.7400584.

(29) Wu, G.; Yussman, M. G.; Barrett, T. J.; Hahn, H. S.; Osinska, H.; Hilliard, G. M.; Wang, X.; Toyokawa, T.; Yatani, A.; Lynch, R. A.; et al. Increased Myocardial Rab GTPase Expression: A Consequence and Cause of Cardiomyopathy. Circ. Res. 2001, 89 (12), 1130-1137. https://doi.org/10.1161/hh2401.100427.

(30) Janka, G. E. Familial and Acquired Hemophagocytic Lymphohistiocytosis. Eur. J. Pediatr. 2007, 166 (2), 95-109. https://doi.org/10.1007/s00431-006-0258-1.

(31) Lin Chua, C. E.; Tang, B. L. $\alpha$ - Synuclein and Parkinson's Disease: The First Roadblock. J. Cell. Mol. Med. 2006, 10 (4), 828-837. https://doi.org/10.2755/jcmm010.004.04.

(32) Donaldson, J.; Segev, N. Regulation and Coordination of Intracellular Trafficking : In Regulation; Springer New York: New York, NY, 2009; pp 329-341. 
https://doi.org/10.1007/978-0-387-93877-6_15.

(33) Lorz, B.; Simson, R.; Nardi, J.; Sackmann, E. Weakly Adhering Vesicles in Shear

Flow: Tanktreading and Anomalous Lift Force. Eur. Lett 2000, 51 (4), 468-474.

https://doi.org/10.1209/ep1/i2000-00517-6.

(34) Skotheim, J. M.; Secomb, T. W. Red Blood Cells and Other Nonspherical Capsules in Shear Flow: Oscillatory Dynamics and the Tank-Treading-to-Tumbling Transition. Phys. Rev. Lett. 2007, 98 (7). https://doi.org/10.1103/PhysRevLett.98.078301.

(35) Kneidl, B.; Peller, M.; Winter, G.; Lindner, L. H.; Hossann, M. Thermosensitive Liposomal Drug Delivery Systems: State of the Art Review. Int. J. Nanomedicine 2014, 9, 4387-4398. https://doi.org/10.2147/IJN.S49297.

(36) Bhatia, T.; Cornelius, F.; Brewer, J.; Bagatolli, L. A.; Simonsen, A. C.; Ipsen, J. H.; Mouritsen, O. G. Spatial Distribution and Activity of Na+/K+-ATPase in Lipid Bilayer Membranes with Phase Boundaries. Biochim. Biophys. Acta-Biomembr. 2016, 1858 (6), 1390-1399. https://doi.org/10.1016/j.bbamem.2016.03.015.

(37) Genova, J.; Ulrih, N.; Kralj-Iglič, V.; Iglič, A.; Bivas, I. Bending Elasticity Modulus of Giant Vesicles Composed of Aeropyrum Pernix K1 Archaeal Lipid. Life 2015, 5 (2), 1101-1110. https://doi.org/10.3390/life5021101.

(38) Bouvrais, H.; Pott, T.; Bagatolli, L. A.; Ipsen, J. H.; Méléard, P. Impact of MembraneAnchored Fluorescent Probes on the Mechanical Properties of Lipid Bilayers. Bba 2010, 1798 (7), 1333-1337. https://doi.org/10.1016/j.bbamem.2010.03.026.

(39) Lei, J.; Glynne-Jones, P.; Hill, M. Acoustic Streaming in the Transducer Plane in Ultrasonic Particle Manipulation Devices. Lab Chip 2013, 13 (11), 2133. https://doi.org/10.1039/c31c00010a.

(40) Sebastian, B.; Favero, T.; Dittrich, P. S. The Effects of Shear Force Transmission Across Vesicle Membranes. J. Phys. Chem. Lett. 2017, 8 (24), 6128-6134. 
https://doi.org/10.1021/acs.jpclett.7b02676.

(41) Yamada, A.; Lee, S.; Bassereau, P.; Baroud, C. N. Trapping and Release of Giant Unilamellar Vesicles in Microfluidic Wells. Soft Matter 2014, 10 (32), 26-28. https://doi.org/10.1039/c4sm00065j.

(42) Pereno, V.; Aron, M.; Vince, O.; Mannaris, C.; Seth, A.; De Saint Victor, M.; Lajoinie, G.; Versluis, M.; Coussios, C.; Carugo, D.; et al. Layered Acoustofluidic Resonators for the Simultaneous Optical and Acoustic Characterisation of Cavitation Dynamics, Microstreaming, and Biological Effects. Biomicrofluidics 2018, 12 (3). https://doi.org/10.1063/1.5023729.

(43) Collis, J.; Manasseh, R.; Liovic, P.; Tho, P.; Ooi, A.; Petkovic-Duran, K.; Zhu, Y. Cavitation Microstreaming and Stress Fields Created by Microbubbles. Ultrasonics 2010, 50 (2), 273-279. https://doi.org/10.1016/j.ultras.2009.10.002.

(44) Portet, T.; Dimova, R. A New Method for Measuring Edge Tensions and Stability of Lipid Bilayers: Effect of Membrane Composition. Biophys. J. 2010, 99 (10), 32643273. https://doi.org/10.1016/j.bpj.2010.09.032.

(45) Rawicz, W.; Smith, B. A.; McIntosh, T. J.; Simon, S. A.; Evans, E. Elasticity, Strength, and Water Permeability of Bilayers That Contain Raft MicrodomainForming Lipids. Biophys. J. 2008, 94 (12), 4725-4736.

https://doi.org/10.1529/biophysj.107.121731.

(46) Keller, S. R.; Skalak, R. Motion of a Tank-Treading Ellipsoidal Particle in a Shear Flow. J. Fluid Mech. 1982, 120 (1), 27-47. https://doi.org/10.1017/S0022112082002651.

(47) Pommella, A.; Brooks, N. J.; Seddon, J. M.; Garbin, V. Selective Flow-Induced Vesicle Rupture to Sort by Membrane Mechanical Properties. Sci Rep 2015, 5, 13163. https://doi.org/10.1038/srep13163. 
(48) Lei, J.; Hill, M.; Glynne-Jones, P. Transducer-Plane Streaming Patterns in Thin-Layer Acoustofluidic Devices. Phys. Rev. Appl. 2017, 8 (1), 014018.

https://doi.org/10.1103/PhysRevApplied.8.014018.

(49) Keren, K.; Yam, P. T.; Kinkhabwala, A.; Mogilner, A.; Theriot, J. A. Intracellular Fluid Flow in Rapidly Moving Cells. Nat. Cell Biol. 2009, 11 (10), 1219-1224. https://doi.org/10.1038/ncb1965.

(50) Moeendarbary, E.; Valon, L.; Fritzsche, M.; Harris, A. R.; Moulding, D. A.; Thrasher, A. J.; Stride, E.; Mahadevan, L.; Charras, G. T. The Cytoplasm of Living Cells Behaves as a Poroelastic Material. https://doi.org/10.1038/nmat3517.

(51) Wang, E.; Goldman, R. D. MOVEMENTS OF CYTOPLASMIC IN BHK-21 IN INTRACELLULAR Time-Lapse Cinernatographic Analysis Statistical Analysis of Particle Movement Cell Cultures Light Microscopy. 1978, 79 (December).

(52) Costaguta, G.; Payne, G. S. Overview of Protein Trafficking Mechanisms. In Trafficking Inside Cells: Pathways, Mechanisms and Regulation; Springer New York: New York, NY, 2009; pp 105-118. https://doi.org/10.1007/978-0-387-93877-6_6.

(53) Davies, P. F. Hemodynamic Shear Stress and the Endothelium in Cardiovascular Pathophysiology. Nature Clinical Practice Cardiovascular Medicine. NIH Public Access 2009, pp 16-26. https://doi.org/10.1038/ncpcardio1397.

(54) Segev, N. Trafficking Inside Cells; 2009. https://doi.org/10.1007/978-0-387-93877-6.

(55) Bjerknes, V. F. K. Fields of Force. Columbio Univ. Press. New York 1906, 1-136.

(56) Versluis, M.; Goertz, D. E.; Palanchon, P.; Heitman, I. L.; Van Der Meer, S. M.; Dollet, B.; De Jong, N.; Lohse, D. Microbubble Shape Oscillations Excited through Ultrasonic Parametric Driving. Phys. Rev. E - Stat. Nonlinear Soft Matter Phys. 2010, 82 (2 Pt 2), 26321. https://doi.org/10.1103/PhysRevE.82.026321.

(57) Martynov, S.; Stride, E.; Saffari, N. The Natural Frequencies of Microbubble 
Oscillation in Elastic Vessels. J. Acoust. Soc. Am. 2009, 126 (6), 2963-2972. https://doi.org/10.1121/1.3243292.

(58) Sebastian, B.; Dittrich, P. ON THE EFFECT OF GEOMETRIC CONSTRAINTS AND MEM-BRANE COMPOSITION ON MECHANOTRANSDUCTION IN GIANT UNILAMELLAR VESICLES.

(59) Vignes, M.; Deleglise, B.; Gougis, P.; Saias, L.; Magnifico, S.; Malaquin, L.; Brugg, B.; Viovy, J.; Peyrin, J. RECONSTRUCTION OF MULTICOMPARTMENT ORIENTED NEURONAL NETWORKS FOR THE STUDY OF NEURODEGENERATIVE DISEASES . 2010, No. October, 43-45.

(60) Noguchi, H.; Gompper, G. Dynamics of Fluid Vesicles in Shear Flow: Effect of Membrane Viscosity and Thermal Fluctuations. Phys. Rev. E - Stat. Nonlinear, Soft Matter Phys. 2005, 72 (1), 11901. https://doi.org/10.1103/PhysRevE.72.011901.

(61) Abkarian, M.; Lartigue, C.; Viallat, A. Tank Treading and Unbinding of Deformable Vesicles in Shear Flow: Determination of the Lift Force. Phys. Rev. Lett. 2002, 88 (6), 68103. https://doi.org/10.1103/PhysRevLett.88.068103.

(62) Pereno, V.; Aron, M.; Vince, O.; Mannaris, C.; Seth, A.; Victor, M. D. Saint; Lajoinie, G.; Versluis, M. Layered Acoustofluidic Resonators for the Simultaneous Optical and Acoustic Characterisation of Cavitation Dynamics, Microstreaming and Biological Effects. Lab a chip [under Rev. 2017.

(63) Pereno, V.; Carugo, D.; Bau, L.; Sezgin, E.; Bernardino De La Serna, J.; Eggeling, C.; Stride, E. Electroformation of Giant Unilamellar Vesicles on Stainless Steel Electrodes. https://doi.org/10.1021/acsomega.6b00395.

(64) Lindken, R.; Rossi, M.; Große, S.; Westerweel, J. Micro-Particle Image Velocimetry (MPIV): Recent Developments, Applications, and Guidelines. Lab Chip 2009, 9 (17), 2551. https://doi.org/10.1039/b906558j. 
(65) Kuhn, P.; Eyer, K.; Robinson, T.; Schmidt, F. I.; Mercer, J.; Dittrich, P. S. A Facile Protocol for the Immobilisation of Vesicles, Virus Particles, Bacteria, and Yeast Cells. Integr. Biol. (Camb). 2012, 4 (12), 1550-1555. https://doi.org/10.1039/c2ib20181j.

(66) Arakawa, T.; Haniu, M.; Narhi, L. O.; Miller, J. A.; Talvenheimo, J.; Philo, J. S.;

Chute, H. T.; Matheson, C.; Carnahan, J.; Louis, J. C.; et al. Formation of Heterodimers from Three Neurotrophins, Nerve Growth Factor, Neurotrophin-3, and Brain-Derived Neurotrophic Factor. J. Biol. Chem. 1994, 269 (45), 27833-27839.

(67) Sennoga, C. a.; Mahue, V.; Loughran, J.; Casey, J.; Seddon, J. M.; Tang, M.;

Eckersley, R. J. On Sizing and Counting of Microbubbles Using Optical Microscopy. Ultrasound Med. Biol. 2010, 36 (12), 2093-2096.

https://doi.org/10.1016/j.ultrasmedbio.2010.09.004.

(68) Chiu, J.-J.; Chien, S. Effects of Disturbed Flow on Vascular Endothelium: Pathophysiological Basis and Clinical Perspectives. Physiol. Rev. 2011, 91 (1), 327387. https://doi.org/10.1152/physrev.00047.2009.

(69) Yang, F.; Gu, N.; Chen, D.; Xi, X.; Zhang, D.; Li, Y.; Wu, J. Experimental Study on Cell Self-Sealing during Sonoporation. J. Control. Release 2008, 131 (3), 205-210. https://doi.org/10.1016/j.jconrel.2008.07.038.

(70) Juffermans, L. J. M.; Kamp, O.; Dijkmans, P. A.; Visser, C. A.; Musters, R. J. P. LowIntensity Ultrasound-Exposed Microbubbles Provoke Local Hyperpolarization of the Cell Membrane Via Activation of BKCa Channels. Ultrasound Med. Biol. 2008, 34 (3), 502-508. https://doi.org/10.1016/j.ultrasmedbio.2007.09.010.

(71) Meijering, B. D. M.; Juffermans, L. J. M.; Van Wamel, A.; Henning, R. H.; Zuhorn, I. S.; Emmer, M.; Versteilen, A. M. G.; Paulus, W. J.; Van Gilst, W. H.; Kooiman, K.; et al. Ultrasound and Microbubble-Targeted Delivery of Macromolecules Is Regulated by Induction of Endocytosis and Pore Formation. Circ. Res. 2009, 104 (5), 679-687. 
https://doi.org/10.1161/CIRCRESAHA.108.183806.

(72) Glynne-Jones, P.; Boltryk, R. J.; Hill, M. Acoustofluidics 9: Modelling and Applications of Planar Resonant Devices for Acoustic Particle Manipulation. Lab Chip 2012, 12 (8), 1417. https://doi.org/10.1039/c2lc21257a.

(73) Nyborg, W. L. Acoustic Streaming near a Boundary. J. Acoust. Soc. Am. 1958, 30 (4), 329-339. https://doi.org/10.1121/1.1909587.

(74) Lee, C. P.; Wang, T. G. Near- boundary Streaming around a Small Sphere Due to Two Orthogonal Standing Waves. J. Acoust. Soc. Am. 1989, 85 (3), 1081-1088. https://doi.org/10.1121/1.397491.

(75) Lei, J.; Hill, M.; Glynne-Jones, P. Numerical Simulation of 3D Boundary-Driven Acoustic Streaming in Microfluidic Devices. Lab Chip 2014, 14 (3), 532-541. https://doi.org/10.1039/c3lc50985k.

(76) Bruus, H. Acoustofluidics 7: The Acoustic Radiation Force on Small Particles. Lab Chip 2012, 12 (6), 1014-1021. https://doi.org/10.1039/c2lc21068a.

(77) Lei, J.; Glynne-Jones, P.; Hill, M. Modal Rayleigh-like Streaming in Layered Acoustofluidic Devices. Phys. Fluids 2016, 28 (1), 012004. https://doi.org/10.1063/1.4939590.

(78) Lei, J. Formation of Inverse Chladni Patterns in Liquids at Microscale: Roles of Acoustic Radiation and Streaming-Induced Drag Forces. Microfluid. Nanofluidics 2017, 21 (3), 50. https://doi.org/10.1007/s10404-017-1888-5.

(79) Lei, J.; Glynne-Jones, P.; Hill, M. Comparing Methods for the Modelling of BoundaryDriven Streaming in Acoustofluidic Devices. Microfluid. Nanofluidics 2017, 21 (2), 23. https://doi.org/10.1007/s10404-017-1865-z. 


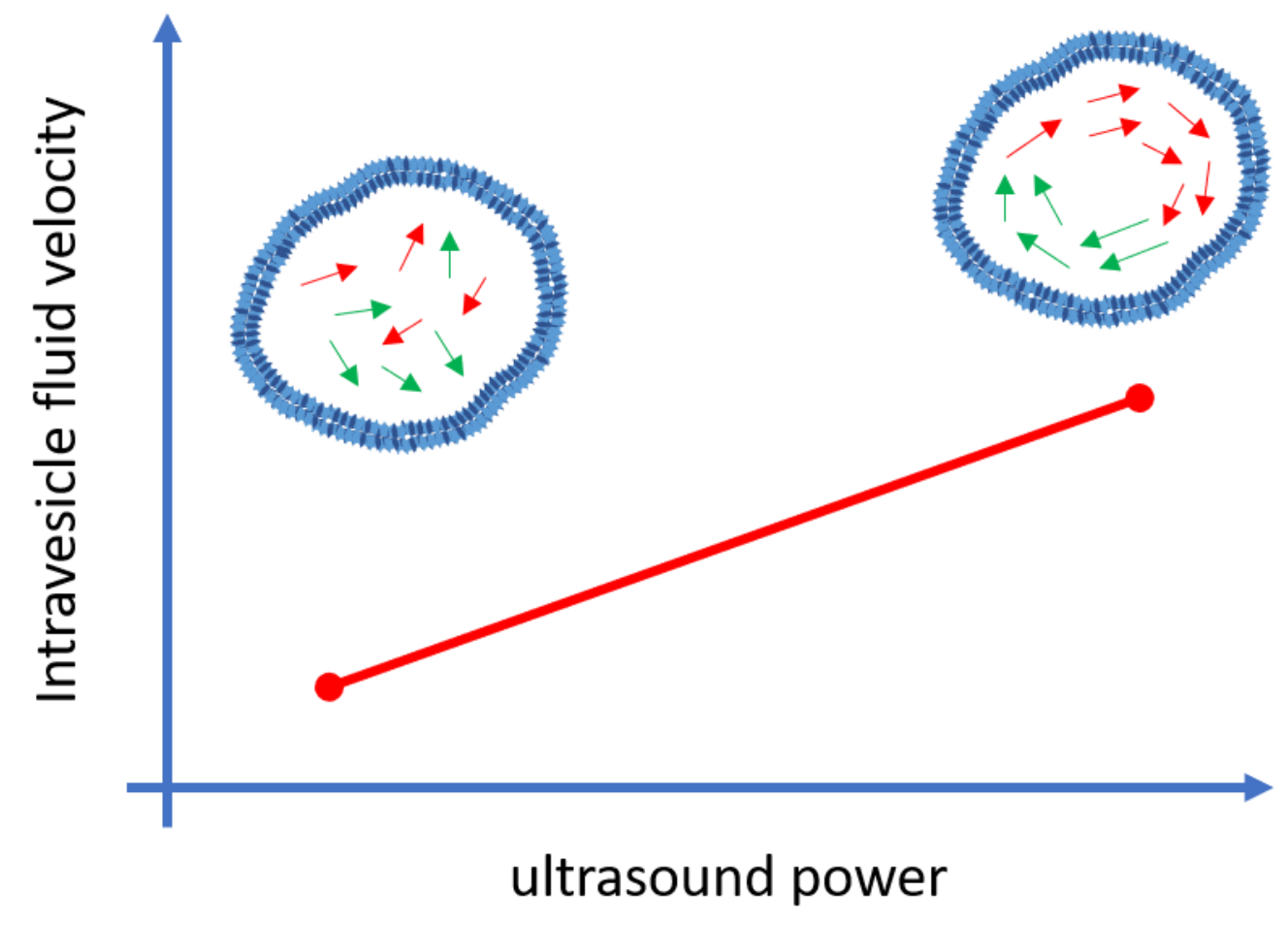

